\title{
NDSC
}

National Disease

Surveillance Centre

\section{Case Definitions}

for Notiffable Diseases

Infectious Diseases (Amendment)

(No. 3) Regulations 2003

(SI No. 707 of 2003) 


\section{Case Definitions for Notifiable Diseases}

Infectious Diseases (Amendment) (No. 3) Regulations 2003 (SI No. 707 of 2003) 


\section{Table of contents}

\section{Introduction}

General Principles for the Application of these Case Definitions

\section{Diseases/pathogens}

Acute anterior poliomyelitis (Polio virus)

Acute infectious gastroenteritis

Ano-genital warts

Anthrax (Bacillus anthracis)

Bacillus cereus food-borne infection/intoxication (B. cereus) 11

Bacterial meningitis (not otherwise specified) 12

Botulism (Clostridium botulinum) 13

Botulism (food-borne) 13

Botulism (infant) 13

Botulism (wound) 13

Botulism (other) $\quad 14$

Brucellosis (Brucella sp.)

Campylobacter infection (Campylobacter sp.) 16

Chancroid (Haemophilus ducreyi) 17

Chlamydia trachomatis infection (genital) (C. trachomatis) 18

Cholera Nibrio cholerae) 19

Clostridium perfringens (type A) food-borne disease (C. perfringens) 20

Creutzfeldt Jakob disease $\quad 21$

Variant Creutzfeldt Jakob disease $\quad 21$

Cryptosporidiosis (Cryptosporidium parvum) 23

Diphtheria (Corynebacterium diphtheriae) $\quad 24$

Echinococcosis (Echinococcus sp.)

Enterococcal bacteraemia (Enterococcus sp. (blood)) 26

Enterohaemorrhagic Escherichia coli (E. coli of serogroup known to be toxin producing) 27

Escherichia coli infection (invasive) (E. coli (blood, CSF)) 28

Giardiasis (Giardia lamblia) $\quad 29$

Gonorrhoea (Neisseria gonorrhoeae) 30

Granuloma inguinale $\quad 31$

Haemophilus influenzae disease (invasive) $(H$. influenzae (blood, CSF or other normally sterile site)) 32

Hepatitis A (acute) (Hepatitis A virus) 33

Hepatitis B (acute and chronic) (Hepatitis B virus) 34

Hepatitis B (acute) $\quad 34$

Hepatitis B (chronic) 34

Hepatitis C (Hepatitis C virus) 35

Herpes simplex (genital) (Herpes simplex virus) 36

Influenza (Influenza A and B virus)

Legionellosis (Legionella sp.) $\quad 38$

Leptospirosis (Leptospira sp.) $\quad 39$ 
Listeriosis (Listeria monocytogenes)

Lymphogranuloma venereum

Malaria (Plasmodium falciparum, vivax, ovale, malariae)

Measles (Measles virus)

Meningococcal Disease (Neisseria meningitidis)

Mumps (Mumps virus)

Non-specific urethritis

Noroviral infection (Norovirus)

Paratyphoid (Salmonella paratyphi) (see typhoid) $\quad 48$

Pertussis (Bordetella pertussis)

Plague (Yersinia pestis)

Q Fever (Coxiella burnetii)

Rabies (Rabies virus)

Rubella (Rubella virus)

Salmonellosis (Salmonella enterica)

Severe Acute Respiratory Syndrome (SARS-associated coronavirus)

Shigellosis (Shigella $s p$.)

Smallpox (Variola virus)

Staphylococcal food poisoning (Enterotoxigenic Staphylococcus aureus)

Staphylococcus aureus bacteraemia (S. aureus (blood))

Streptococcus group A infection (invasive) (S. pyogenes (blood, CSF or other normally sterile site))

Streptococcus pneumoniae infection (invasive) (S. pneumoniae (blood, CSF or other normally sterile site))

Syphilis (Treponema pallidum)

Tetanus (Clostridium tetani)

Toxoplasmosis (Toxoplasma gondii)

Trichinosis (Trichinella sp.)

Trichomoniasis (Trichomonas vaginalis) $\quad 73$

Tuberculosis (Mycobacterium tuberculosis complex) 74

Tularemia (Francisella tularensis) $\quad 75$

Typhoid/paratyphoid fever (Salmonella typhi/paratyphi) 76

Typhus (Rickettsia prowazekii) $\quad 77$

Viral encephalitis $\quad 78$

Viral haemorrhagic fevers (Lassa virus, Marburg virus, Ebola virus,

Crimean-Congo haemorrhagic fever virus)

Lassa fever

Ebola/Marburg disease

Crimean-Congo haemorrhagic fever $\quad 80$

Viral meningitis

Yellow fever (Yellow Fever virus)

Yersiniosis (Yersinia enterocolitica, Yersinia pseudotuberculosis)

Definition of Outbreak

Definition of Unusual Cluster or Changing Pattern of Illness $\quad 85$

Appendix 1. List of Diseases

Appendix 2. Members of the Sub-Committee of the Scientific Advisory Committee 


\section{Introduction}

The Infectious Diseases Regulations 1981, as most recently amended by the Infectious Diseases (Amendment) (No. 3) Regulations 2003 (SI No. 707 of 2003), requires that a medical practitioner and a clinical director of a diagnostic laboratory, in notifying infectious diseases, shall have regard to the case definitions for infectious diseases circulated by the National Disease Surveillance Centre. A case definition means the set of clinical or microbiological characteristics by which a case of infectious disease is defined. The NDSC shall be responsible for maintaining, updating and circulating the case definitions. This document sets out the case definitions for the diseases listed in the Schedule to the aforementioned Regulations. (Appendix 1)

A Decision of the European Commission (Decision No. 2002/253/EC) specifies the case definitions to be applied by Member States for the purposes of submitting data for the epidemiological surveillance and control of communicable diseases under the provisions of Decision No. 2119/98/EC.

In this document, the standardised European case definitions mentioned above are used where possible. Where these definitions are used, EU appears in brackets after the heading. Where European definitions are not available, Centers for Disease Control and Prevention (CDC), Laboratory Centre for Disease Control (Canada) (LCDC) or World Health Organisation (WHO) definitions were used or adapted. Where existing Irish definitions were already available, the National Expert Groups responsible for those definitions were consulted and in some cases the Irish definitions are used. Data on invasive staphylococcal and Escherichia coli infections are collected as part of the European Antimicrobial Surveillance System (EARSS) and EARSS definitions are used for these pathogens.

This document is based on the two reports produced by the Notifiable Diseases Sub-Committee of the Scientific Advisory Committee of the National Disease Surveillance Centre (see Appendix 2 for membership) entitled Review of Notifiable Diseases and the Process of Notification (February 2001) and Case definitions, Accompanying Document to the Review of Notifiable Diseases and the Process of Notification (November 2002).

The changes brought about by the Infectious Diseases (Amendment) (No. 3) Regulations 2003 (SI No. 707 of 2003) came into effect on 1 January 2004. It is expected that, with use in practice, some of the case definitions may be amended over time; the current version may be found at all times on the website of the NDSC (www.ndsc.ie). 


\section{General Principles for the Application of these Case Definitions}

1. Unless specifically stated, only symptomatic cases are to be reported. Asymptomatic infections are to be regarded as cases, however, if the infection has therapeutic or public health implications.

A "case with an epidemiological link" is a case that has either been exposed to a confirmed case, or has had the same exposure as a confirmed case (e.g. eaten the same food, stayed in the same hotel, etc).

2. A 3-tiered system with the following levels is used:

- Possible case: indicative clinical picture without being a confirmed or probable case

- Probable case: clear clinical picture, or linked epidemiologically to a confirmed case;

- Confirmed case: verified by laboratory analysis.

The classification on these different levels might vary according to the epidemiology of the individual diseases.

3. Clinical symptoms listed are only given as indicative examples, and are not exhaustive. The clinical description gives a general outline of the disease and does not necessarily indicate all the features needed for clinical diagnosis of the disease.

4. For most diseases, several 'criteria for laboratory diagnosis' are listed. Unless otherwise stated, only one of these is needed to confirm a case.

5. In Ireland a specific antibody response is interpreted as a four-fold rise in titre.

6. N/A in the case definition list means 'not applicable'. 


\section{Acute anterior poliomyelitis (Polio virus) (EU)}

\section{Clinical description}

Clinical picture compatible with poliomyelitis, i.e. acute onset of a flaccid paralysis of one or more limbs with decreased or absent tendon reflexes in the affected limbs, without other apparent cause, and without sensory or cognitive loss.

\section{Laboratory criteria for diagnosis}

One of the following:

- Detection of polio virus nucleic acid

- Isolation of polio virus from a clinical specimen

\section{Case classification}

Possible:

Probable:

Confirmed:

\section{$\mathrm{N} / \mathrm{A}$}

A case that meets the clinical case definition

A case that meets the clinical case definition and is laboratory confirmed. 


\section{Acute infectious gastroenteritis}

\section{Case classification}

Possible: N/A

Probable: $\quad$ Acute onset of diarrhoea and/or vomiting with no known noninfectious cause.

Confirmed: If a laboratory diagnosis has been made see definition for specific organism.

\section{Note:}

Rotavirus, although not specifically listed, should be reported under the category of acute infectious gastroenteritis. 


\section{Ano-genital warts}

\section{Clinical description}

An infection characterised by the presence of visible, raised growths on the internal or external genitalia, perineum, or perianal region.

\section{Laboratory criteria for diagnosis}

One of the following:

- Histopathological confirmation.

- Detection of human papilloma virus DNA.

\section{Case classification}

\section{Possible: N/A}

Probable: A clinically compatible case without histopathologic diagnosis and without microscopic or serologic evidence that the growth is the result of secondary syphilis

Confirmed: A clinically compatible case that is laboratory confirmed. 


\section{Anthrax \\ (Bacillus anthracis) (EU)}

\section{Clinical description}

Inhalational anthrax

Following inhalation of $B$. anthracis and a brief prodrome, acute febrile respiratory failure develops with hypoxia, dyspnoea, and radiological evidence of mediastinal widening.

\section{Cutaneous anthrax}

A skin lesion evolving from a papule, through a vesicular stage to a depressed black eschar with surrounding oedema. The lesion is usually painless but there may be constitutional disturbance (fever and malaise).

\section{Gastrointestinal anthrax}

Following consumption of raw contaminated food, a syndrome of severe abdominal pain, diarrhoea, fever and septicaemia

\section{Laboratory criteria for diagnosis}

One of the following:

- Isolation and confirmation of $B$. anthracis from specimens collected from a normally sterile site (e.g. blood or cerebrospinal fluid) or lesion of other affected tissue (skin, lung or gut)

- Both of the following

o evidence of $B$. anthracis DNA (e.g. by PCR) from specimens collected from a normally sterile site (e.g. blood or cerebrospinal fluid) or lesion of other affected tissue (skin, lung or gut)

o demonstration of $B$. anthracis in a clinical specimen by immunohistochemical staining of affected tissue (skin, lung or gut)

Nasal swab without indication of disease does not contribute to diagnosis of a case.

\section{Case classification}

Possible: N/A

Probable: One of the following:

- A clinically compatible case of illness without isolation of $B$. anthracis and no alternative diagnosis, but with laboratory evidence of $B$. anthracis by one supportive laboratory test 
- A clinically compatible case of anthrax epidemiologically linked* to a confirmed environmental exposure, but without corroborative laboratory evidence of $B$. anthracis infection

Confirmed: A clinically confirmed case that is laboratory confirmed.

* See General Principles for the Application of these Case Definitions page 5 


\section{Bacillus cereus food-borne infection/intoxication \\ (B. cereus)}

\section{Clinical description}

Clinical picture compatible with $B$. cereus gastrointestinal infection or intoxication characterised in some cases by sudden onset of nausea and vomiting, and in others by abdominal cramps and diarrhoea.

\section{Laboratory criteria for diagnosis}

One of the following:

- Isolation of $10^{5} \mathrm{~B}$. cereus organisms per gram or direct detection of

B. cereus enterotoxin from epidemiologically implicated food in the setting of a person or persons with diarrhoea or vomiting

- Isolation of the organism from the stools of two or more ill persons but not from the stools of controls, in an outbreak situation

\section{Case classification}

Possible: N/A

Probable: A clinically compatible case with an epidemiological link*

Confirmed: A case that is laboratory confirmed.

* See General Principles for the Application of these Case Definitions page 5 


\section{Bacterial meningitis (not otherwise specified)}

\section{Clinical description}

Bacterial meningitis manifests most commonly with fever, headache, and a stiff neck; the disease may progress rapidly to shock and death. However, other manifestations may be observed.

\section{Laboratory criteria for diagnosis}

Isolation of a bacterial species from the cerebrospinal fluid

\section{Case classification}

Possible: A clinically compatible case

Probable: Laboratory evidence of bacterial meningitis but culture negative Confirmed: Isolation of a bacterial species from the cerebrospinal fluid. If a diagnosis of meningitis due to Haemophilus influenzae, meningococcus, pneumococcus or other listed bacterial pathogen has been made, see definition for particular organism. 


\section{Botulism \\ (Clostridium botulinum)}

\section{Botulism (food-borne) (EU)}

\section{Clinical description}

Clinical picture compatible with botulism, i.e. symptoms such as diplopia,

blurred vision or bulbar weakness. Symmetric paralysis may progress rapidly.

\section{Laboratory criteria for diagnosis}

One of the following:

- Detection of botulinum toxin in serum, stool, stomach content or patient's food

- Isolation of $C$. botulinum from stool

\section{Case classification}

Possible: N/A

Probable: A clinically compatible case with an epidemiological link*

Confirmed: A clinically compatible case that is laboratory confirmed

\section{Botulism (infant)}

\section{Clinical description}

An illness of infants, characterised by constipation, poor feeding, and "failure to thrive" that may be followed by progressive weakness, impaired respiration, and death

\section{Laboratory criteria for diagnosis}

One of the following:

- Detection of botulinum toxin in stool or serum

- Isolation of $C$. botulinum from stool

\section{Case classification}

Possible: N/A

Probable: N/A

Confirmed: A clinically compatible case that is laboratory-confirmed, occurring in a child aged $<1$ year.

* See General Principles for the Application of these Case Definitions page 5 


\section{Botulism (wound)}

\section{Clinical description}

An illness resulting from toxin produced by $C$. botulinum that has infected a wound. Common symptoms are diplopia, blurred vision, and bulbar weakness. Symmetric paralysis may progress rapidly.

\section{Laboratory criteria for diagnosis}

One of the following:

- Detection of botulinum toxin in serum

- Isolation of $C$. botulinum from wound

\section{Case classification}

Possible: N/A

Probable: N/A

Confirmed: A clinically compatible case that is laboratory confirmed in a patient who has no suspected exposure to contaminated food and who has a history of a fresh, contaminated wound during the 2 weeks before onset of symptoms.

\section{Botulism (other)}

\section{Clinical description}

Clinical picture compatible with botulism, i.e. symptoms such as diplopia, blurred vision or bulbar weakness. Symmetric paralysis may progress rapidly.

\section{Laboratory criteria for diagnosis}

One of the following:

- Detection of botulinum toxin in clinical specimen

- Isolation of $C$. botulinum from clinical specimen

\section{Case classification}

Possible: N/A

Probable: N/A

Confirmed: A clinically compatible case that is laboratory confirmed in a patient aged 1 year or over who has no history of ingestion of suspect food and has no wounds.

* See General Principles for the Application of these Case Definitions page 5 


\section{Brucellosis}

\section{(Brucella sp.) (EU)}

\section{Clinical description}

Clinical picture compatible with brucellosis i.e. acute or insidious onset of fever, night sweats, undue fatigue, anorexia, weight loss, headache, or arthralgia.

\section{Laboratory criteria for diagnosis}

One of the following:

- Demonstration of a specific antibody response (four fold or greater rise)

- Demonstration by immunofluorescence of Brucella sp. in a clinical specimen

- Isolation of Brucella sp. from a clinical specimen

For probable case

A single high titre

\section{Case classification}

Possible: N/A

Probable: A clinically compatible case with an epidemiological link, ${ }^{*}$ or a case with an isolated high titre

Confirmed: A clinically compatible case that is laboratory confirmed.

* See General Principles for the Application of these Case Definitions page 5 


\section{Campylobacter infection \\ (Campylobacter sp.) (EU)}

\section{Clinical description}

Clinical picture compatible with campylobacter infection, i.e. an illness with diarrhoea and/ or vomiting of variable severity which may be accompanied by abdominal cramps and fever ${ }^{\star \star}$. Cases may also be asymptomatic.

\section{Laboratory criteria for diagnosis}

Isolation of Campylobacter sp. from any clinical specimen

\section{Case classification}

Possible: N/A

Probable: A clinically compatible case with an epidemiological link*

Confirmed: A clinically compatible case (symptomatic or asymptomatic) that is laboratory confirmed.

** The description in the EU case definition document has been elaborated upon here

* See General Principles for the Application of these Case Definitions page 5 


\section{Chancroid}

\section{(Haemophilus ducreyi)}

\section{Clinical description}

A sexually transmitted disease characterized by painful genital ulceration and inflammatory inguinal adenopathy. The disease is caused by infection with Haemophilus ducreyi.

\section{Laboratory criteria for diagnosis}

Isolation of $H$. ducreyi from a clinical specimen

\section{Case classification}

Possible: N/A

Probable: A clinically compatible case with both a) no evidence of Treponema pallidum infection by darkfield microscopic examination of ulcer exudate or by a serologic test for syphilis performed greater than or equal to 7 days after onset of ulcers and b) either a clinical presentation of the ulcer(s) not typical of disease caused by herpes simplex virus (HSV) or a culture negative for HSV.

Confirmed: A clinically compatible case that is laboratory confirmed. 


\section{Chlamydia trachomatis infection (genital) \\ (C. trachomatis) (EU)}

\section{Clinical description}

Clinical picture compatible with C. trachomatis infection, e.g. urethritis, epididymitis, cervicitis, acute salpingitis or other syndromes when sexually transmitted.

\section{Laboratory criteria for diagnosis}

One of the following:

- Isolation of $C$. trachomatis by culture from specimen of the uro-genital tract

- Demonstration of $C$. trachomatis in a clinical specimen from the uro-genital tract by detection of antigen or nucleic acid.

\section{Case classification}

Possible: N/A

Probable: N/A

Confirmed: A case that is laboratory confirmed. 


\section{Cholera}

\section{(Vibrio cholerae) (EU)}

\section{Clinical description}

Clinical picture compatible with cholera i.e. watery diarrhoea and/or vomiting. Severity is variable.

\section{Laboratory criteria for diagnosis}

One of the following:

- Isolation of toxigenic (i.e. cholera toxin-producing) V. cholerae $\mathrm{O} 1$ or 0139 from stool or vomitus

- Demonstration of a specific antitoxin and vibrocidal antibody response.

\section{Case classification}

Possible: N/A

Probable: A clinically compatible case with an epidemiological link* Confirmed: A clinically compatible case that is laboratory confirmed.

* See General Principles for the Application of these Case Definitions page 5 


\section{Clostridium perfringens (type A) food-borne disease \\ (C. perfringens)}

\section{Clinical description}

Clinical picture compatible with an intestinal disorder, characterised by sudden onset of abdominal cramps followed by diarrhoea, with or without nausea.

\section{Laboratory criteria for diagnosis}

One of the following:

- One or more of the following in the above clinical setting:

o Detection of $10^{5}$ colony-forming units of $C$. perfringens per gram of epidemiologically implicated food

o Direct detection of $C$. perfringens enterotoxin from faeces

- Demonstration of $10^{6} \mathrm{C}$. perfringens spores per gram of faeces in a specimen taken within 48 hours of the onset of symptoms consistent with food-borne $C$. perfringens disease in an outbreak setting

\section{Case classification}

Possible: N/A

Probable: A clinically compatible case with an epidemiological link*

Confirmed: A case that is laboratory confirmed. 


\section{Creutzfeldt Jakob disease}

\section{Case classification}

Possible: All of the following:

- Progressive dementia; and

- EEG atypical or not known and

- Duration <two years

and

- at least 2 out of the following four clinical features: myoclonus, visual or cerebellar disturbance, pyramidal/extrapyramidal dysfunction, akinetic mutism

Probable: (in the absence of an alternative diagnosis from routine investigation)

- Progressive dementia and

- At least 2 of the following 4 clinical features: myoclonus, visual or cerebellar disturbance, pyramidal/extrapyramidal dysfunction, akinetic mutism

and

- A typical EEG, whatever the clinical duration of the disease and/or

- A positive 14-3-3 assay for CSF and a clinical duration to death $<2$ years

Confirmed: • Neuropathological confirmation and/or

- Confirmation of protease-resistant prion protein (PrP) (immunocytochemistry or Western blot) and/or

- Presence of scrapie-associated fibrils.

\section{Variant Creutzfeldt Jakob disease (EU)}

\section{Clinical description}

\section{History}

- Progressive neuropsychiatric disorder

- Duration of illness > 6 months

- Routine investigations do not suggest an alternative diagnosis

- No history of potential iatrogenic exposure 


\section{Clinical features}

- Early psychiatric symptoms

- Persistent painful sensory symptoms

- Ataxia

- Myoclonus or chorea or dystonia

- Dementia

\section{Laboratory criteria for diagnosis}

(This section has been expanded to include other specific non-laboratory based tests)

- EEG does not show typical appearance of classical CJD (or no EEG performed),

- Bilateral pulvinar high signal on MRI scan,

- Characteristic neuropathological and immunopathological findings.

\section{Case classification}

Possible: N/A

Probable: I and 4/5 of clinical features, and EEG does not show typical appearance of classical CJD (or no EEG performed) and Bilateral pulvinar high signal on MRI scan I and positive tonsil biopsy

Confirmed: Progressive neuropsychiatric disorder and neuropathological confirmation of diagnosis of vCJD.

\section{Note:}

Confirmed cases of Creutzfeldt Jakob disease and variant Creutzfeldt Jakob disease are notifiable. Notifiers are also requested to report possible and probable cases to the Medical Officer of Health. 


\section{Cryptosporidiosis \\ (Cryptosporidium parvum) (EU)}

\section{Clinical description}

Clinical picture compatible with cryptosporidiosis, characterised by diarrhoea, abdominal cramps, loss of appetite, nausea and vomiting.

\section{Laboratory criteria for diagnosis}

One of the following:

- Demonstration of cryptosporidium oocysts in stool

- Demonstration of Cryptosporidium sp. in intestinal fluid or small-bowel biopsy specimens

- Demonstration of cryptosporidium antigen in stool

\section{Case classification}

Possible: N/A

Probable: A clinically compatible case with an epidemiological link*

Confirmed: A case that is laboratory confirmed.

* See General Principles for the Application of these Case Definitions page 5 


\section{Diphtheria \\ (Corynebacterium diphtheriae) (EU)}

\section{Clinical description}

Clinical picture compatible with either respiratory diphtheria, i.e. an upper respiratory tract illness characterised by an adherent membrane of the tonsils, pharynx or nose, in combination with sore throat and low grade fever, or nonrespiratory diphtheria, i.e. an illness characterised by cutaneous, conjunctival, otic, genital or other types of ulcers.

\section{Laboratory criteria for diagnosis}

Isolation of diphtheria toxin-producing corynebacteria (typically

Corynebacterium diphtheriae or C. ulcerans) from a clinical specimen.

\section{Case classification}

Possible:

$\mathrm{N} / \mathrm{A}$

Probable:

A clinically compatible case

Asymptomatic carriers: Asymptomatic carriers with toxigenic strains Confirmed: A clinically compatible case that is laboratory confirmed with the isolation of a toxigenic strain of corynebacteria, or a clinically compatible case with an epidemiological link ${ }^{*}$ to a laboratory confirmed case.

It is to be noted that both respiratory and non-respiratory diphtheria cases with isolation of toxigenic strains should be reported, as should asymptomatic carriers with toxigenic strains, if they are detected. Cases with non-toxigenic C. diphtheriae or C. ulcerans should not be reported.

* See General Principles for the Application of these Case Definitions page 5 


\section{Echinococcosis}

\section{(Echinococcus sp.) (EU)}

\section{Clinical description}

Clinical picture compatible with echinococcosis, which may produce any of several clinical syndromes, varying with cyst size and location.

\section{Laboratory criteria for diagnosis}

One of the following:

- Histopathology

- A combination of imaging techniques and serological tests (e.g. indirect haemagglutination, immunodiffusion, immunoblot assay)

\section{Case classification}

\section{Possible: N/A}

Probable: N/A

Confirmed: A clinically compatible case that is laboratory confirmed. 


\section{Enterococcal bacteraemia}

\section{(Enterococcus sp. (blood))}

\section{Laboratory criteria for diagnosis}

Isolation of Enterococcus sp. from blood. Susceptibility to vancomycin or teicoplanin, ampicillin and gentamicin (high level) should be determined.

\section{Case classification}

Possible: N/A

Probable: N/A

Confirmed: A case that is laboratory confirmed. 


\section{Enterohaemorrhagic Escherichia coli \\ (E. coli of serogroup known to be toxin producing) \\ (EU)}

\section{Clinical description}

Clinical picture compatible with enterohaemorrhagic E. coli infection, e.g. diarrhoea (often bloody) and abdominal cramps. Illness may be complicated by haemolytic uraemic syndrome (HUS) or thrombotic thrombocytopenic purpura (TTP). Cases may also be asymptomatic ${ }^{\star \star}$.

\section{Laboratory criteria for diagnosis}

One of the following:

- Isolation of E. coli belonging to a sero-group known to cause enterohaemorrhagic disease

- Serological confirmation in patients with HUS or TTP

- For probable cases: detection of genes coding for Stx1/Stx2 production

\section{Case classification}

Possible: N/A

Probable: A laboratory confirmed isolate without clinical information or a case with clinical symptoms that has an epidemiological link*

Confirmed: A clinically compatible case that is laboratory confirmed.

* See General Principles for the Application of these Case Definitions page 5

** The description in the EU case definition document has been elaborated upon here. 


\section{Escherichia coli infection (invasive)}

(E. coli (blood, CSF))

\section{Laboratory criteria for diagnosis}

Isolation of E. coli from blood or cerebrospinal fluid. Susceptibility to ampicillin, third generation cephalosporins, gentamicin and ciprofloxacin should be determined.

\section{Case classification}

Possible: N/A

Probable: N/A

Confirmed: A case that is laboratory confirmed, 


\section{Giardiasis}

(Giardia lamblia) (EU)

\section{Clinical description}

Clinical picture compatible with infection with G. lamblia, characterised by diarrhoea, abdominal cramps, bloating, weight loss, or malabsorption.

\section{Laboratory criteria for diagnosis}

One of the following:

- Demonstration of G. lamblia cysts in stool

- Demonstration of G. lamblia trophozoites in stool, duodenal fluid, or smallbowel biopsy

- Demonstration of G. lamblia antigen in stool

\section{Case classification}

Possible: N/A

Probable: A clinically compatible case that has an epidemiological link ${ }^{*}$

Confirmed: A case that is laboratory confirmed.

* See General Principles for the Application of these Case Definitions page 5 


\section{Gonorrhoea \\ (Neisseria gonorrhoeae) (EU)}

\section{Clinical description}

A sexually transmitted infection commonly manifested by urethritis, cervicitis or salpingitis and occasionally by proctitis, pharyngitis or tonsillitis. Infection may also be asymptomatic. ${ }^{* *}$

\section{Laboratory criteria for diagnosis}

One of the following:

- Isolation of $N$. gonorrhoeae from a clinical specimen

- Demonstration of $N$. gonorrhoeae antigen or nucleic acid

- Demonstration of gram negative intracellular diplococci in a urethral smear from a male

\section{Case classification}

Possible: N/A

Probable: N/A

Confirmed: A case that is laboratory confirmed.

** The description in the EU case definition document has been elaborated upon here. 


\section{Granuloma inguinale}

\section{Clinical description}

A slowly progressive ulcerative disease of the skin and lymphatics of the genital and perianal area caused by infection with Calymmatobacterium granulomatis. A clinically compatible case would have one or more painless or minimally painful granulomatous lesions in the anogenital area.

\section{Laboratory criteria for diagnosis}

Demonstration of intracytoplasmic Donovan bodies in Wright or Giemsastained smears or biopsies of granulation tissue

\section{Case classification}

Possible: N/A

Probable: A clinically compatible case

Confirmed: A clinically compatible case that is laboratory confirmed. 


\section{Haemophilus influenzae disease (invasive) \\ (H. influenzae (blood, CSF or other normally sterile site)) (EU)}

\section{Clinical description}

Clinical picture compatible with invasive disease, i.e. bacteraemia, meningitis, arthritis, epiglottitis, osteomyelitis or cellulitis.

\section{Laboratory criteria for diagnosis}

One of the following:

- Isolation of $H$. influenzae from normally sterile site

- Detection of $H$. influenzae nucleic acid from a normally sterile site

For probable case

- Detection of $H$. influenzae antigen from a normally sterile site

\section{Case classification}

Possible: A case with clinical epiglottitis without any laboratory confirmation or with identification only from non-sterile site

Probable: A clinically compatible case with antigen detection as above Confirmed: A clinically compatible case that is laboratory confirmed. 


\section{Hepatitis A (acute) \\ (Hepatitis A virus) (EU)}

\section{Clinical description}

In symptomatic cases, clinical picture compatible with hepatitis, i.e. discrete onset of symptoms and/or jaundice or elevated serum aminotransferase levels. Asymptomatic cases are common.*

\section{Laboratory criteria for diagnosis}

One of the following:

- IgM-class to hepatitis A virus (anti-HAV) positive

- Detection of antigen in stool

- Detection of nucleic acid in serum

\section{Case classification}

Possible: A case that meets the clinical case definition but has no epidemiological link ${ }^{*}$

Probable: A case that meets the clinical case definition and has an epidemiological link*

Confirmed: A case that meets the clinical case definition and is laboratory confirmed.

** The description in the EU case definition document has been elaborated upon here

* See General Principles for the Application of these Case Definitions page 5 


\section{Hepatitis B (acute and chronic) (Hepatitis B virus)}

\section{Clinical description}

In symptomatic cases, clinical picture compatible with hepatitis, i.e. discrete onset of symptoms and/or jaundice or elevated serum aminotransferase levels. Asymptomatic cases are common. ${ }^{* *}$

\section{Hepatitis B (acute) (EU)}

\section{Laboratory criteria for diagnosis}

One of the following:

- IgM antibody to hepatitis B core antigen (anti-HBc) positive

- Detection of hepatitis B virus (HBV) nucleic acid in serum

\section{Case classification}

Possible: N/A

Probable: A symptomatic case that is HBsAg positive and has a clinical picture compatible with an acute hepatitis

Confirmed: A case that is laboratory confirmed

\section{Hepatitis B (chronic)}

\section{Laboratory criteria for diagnosis}

One of the following:

- Hepatitis B surface antigen (HBsAg) positive and antibody to hepatitis B core antigen (anti-HBc) positive and IgM antibody to hepatitis B core antigen negative

- Persistence for more than 6 months of either HBsAg or HBV nucleic acid in serum

\section{Case classification}

Possible: N/A

Probable: N/A

Confirmed: A case that is laboratory confirmed.

\section{Note:}

In notifying a case of hepatitis B, indicate whether acute or chronic.

Notify chronic cases unless known to have been previously notified.

** The description in the EU case definition document has been elaborated upon here 


\section{Hepatitis C \\ (Hepatitis C virus) (EU)}

\section{Clinical description}

In symptomatic cases, clinical picture compatible with hepatitis, i.e. discrete onset of symptoms and/or jaundice or elevated serum aminotransferase levels. Asymptomatic cases are common. ${ }^{* *}$

\section{Laboratory criteria for diagnosis}

One of the following:

- Detection of hepatitis C virus (HCV) specific antibodies

- Detection of HCV nucleic acid from clinical samples

\section{Case classification}

Possible: N/A

Probable: N/A

Confirmed: A case that is laboratory confirmed.

\section{Note:}

This case definition includes all laboratory confirmed cases; the EU definition is restricted to symptomatic cases only.

** The description in the EU case definition document has been elaborated upon here 


\section{Herpes simplex (genital) \\ (Herpes simplex virus)}

\section{Clinical description}

A condition characterised by visible, painful genital or anal lesions. Clinical presentation may be atypical.

\section{Laboratory criteria for diagnosis}

One of the following:

- Isolation of herpes simplex virus (HSV) from cervix, urethra, or anogenital lesion

- Demonstration of virus by antigen detection technique in clinical specimens from cervix, urethra, or anogenital lesion

- Demonstration of multinucleated giant cells on a Tzanck smear of scrapings from an anogenital lesion

- Serological type specific antibody to HSV type 1 or type 2 virus.

\section{Case classification}

Possible: N/A

Probable: A clinically compatible case (in which primary and secondary syphilis have been excluded by appropriate serologic tests and darkfield microscopy, when available) with either a diagnosis of genital herpes based on clinical presentation (without laboratory confirmation) or a history of one or more previous episodes of similar genital lesions

Confirmed: A clinically compatible case, including an atypical presentation, that is laboratory confirmed. 


\section{Influenza}

\section{(Influenza A and B virus) (EU)}

\section{Clinical description}

Clinical picture compatible with influenza i.e. sudden onset of disease, cough, fever $>38^{\circ} \mathrm{C}$, muscular pain and/or headache.

\section{Laboratory criteria for diagnosis}

One of the following:

- Detection of influenza antigen, or influenza virus specific RNA

- Isolation of influenza virus

- Demonstration of a specific serum antibody response to influenza A or B (four fold or greater rise). ${ }^{* \star}$

\section{Case classification}

Possible: Clinically compatible case with epidemiological link*

Probable: N/A

Confirmed: Clinical case that is laboratory confirmed.

\section{Note:}

Influenza of a new or re-emergent subtype is defined as influenza caused by a novel or re-emerging influenza virus which is capable of infecting humans and causing human illness, and to which a large proportion of the human population has little or no immunity.

**The description in the EU case definition document has been elaborated upon here.

* See General Principles for the Application of these Case Definitions page 6 


\section{Legionellosis \\ (Legionella sp.) (EU)}

\section{Clinical description}

\section{Legionnaires' Disease}

Pneumonia

\section{Pontiac fever}

A self limiting influenza like illness characterised by fever, headache, myalgia and non-productive cough. Patients recover spontaneously without therapy after 2 to 5 days. No signs of pneumonia.

\section{Laboratory criteria for diagnosis}

One of the following:

- Isolation of any legionella organism from respiratory secretions, lung tissue or blood

- Demonstration of a specific antibody response (four fold or greater rise*) to L. pneumophila serogroup 1 or other serogroups or other Legionella species by the indirect immunofluorescent antibody test or by microagglutination

- The detection of specific legionella antigen in urine using validated reagents

\section{For probable case}

One of the following:

- A single high titre in specific serum antibody to L. pneumophila serogroup 1, other serogroups or other Legionella species

- Detection of specific legionella antigen in respiratory secretions or direct fluorescent antibody (DFA) staining of the organism in respiratory secretions or lung tissue using evaluated monoclonal reagents

\section{Case classification}

Possible: N/A

Probable: A clinically compatible case that is tested by laboratory as probable (see above) or a clinically compatible case with an epidemiological link*

Confirmed: A clinically compatible case that is laboratory confirmed.

* See General Principles for the Application of these Case Definitions page 5 


\section{Leptospirosis \\ (Leptospira sp.) (EU)}

\section{Clinical description}

Clinical picture compatible with leptospirosis, characterised by fever, headache, chills, myalgia, conjunctival suffusion, and less frequently by

meningitis, rash, jaundice, or renal insufficiency.

\section{Laboratory criteria for diagnosis}

One of the following:

- Isolation of Leptospira sp. from a clinical specimen

- Demonstration of specific rise in leptospira agglutination titre (four fold or greater rise) $^{\star}$

- Demonstration of Leptospira sp. in a clinical specimen by immunofluorescence

- Detection of Leptospira IgM antibody in serum

\section{Case classification}

Possible: N/A

Probable: N/A

Confirmed: A clinically compatible case that is laboratory confirmed.

* See General Principles for the Application of these Case Definitions page 5 


\section{Listeriosis}

\section{(Listeria monocytogenes) (EU)}

\section{Clinical description}

Infection caused by L. monocytogenes, which may produce any of several clinical syndromes, including stillbirth, listeriosis of the newborn, meningitis, bacteraemia or localised infections.

\section{Laboratory criteria for diagnosis}

Isolation of L. monocytogenes from a normally sterile site (e.g. blood or cerebrospinal fluid or, less commonly, joint, pleural or pericardial fluid)

\section{Case classification}

Possible: N/A

Probable: $\quad \mathrm{N} / \mathrm{A}$

Confirmed: A clinically compatible case that is laboratory confirmed. 


\section{Lymphogranuloma venereum}

\section{Clinical description}

Infection with L1, L2, or, L3 serovars of Chlamydia trachomatis may result in a disease characterised by genital lesions, suppurative regional lymphadenopathy, or hemorrhagic proctitis. The infection is usually sexually transmitted.

\section{Laboratory criteria for diagnosis}

One of the following:

- Isolation of C. trachomatis, serotype L1, L2, or L3 from clinical specimen

- Demonstration by immunofluorescence of inclusion bodies in leukocytes of an inguinal lymph node (bubo) aspirate

- Positive microimmunofluorescent serologic test for a lymphogranuloma venereum strain of $C$. trachomatis

\section{Case classification}

Possible: N/A

Probable: A clinically compatible case with one or more tender fluctuant inguinal lymph nodes or characteristic proctogenital lesions with supportive laboratory findings of a single $C$. trachomatis complement fixation titre of $>64$

Confirmed: A clinically compatible case that is laboratory confirmed. 


\section{Malaria}

(Plasmodium falciparum, vivax, ovale, malariae) (EU)

\section{Clinical description}

Clinical picture compatible with malaria, i.e. fever and commonly associated symptoms, which include headache, back pain, chills, sweats, myalgia, nausea, vomiting, diarrhoea and cough.

\section{Laboratory criteria for diagnosis}

One of the following:

- Demonstration of malaria parasites in blood films

- Detection of plasmodium nucleic acid

\section{Case classification}

Possible: N/A

Probable: N/A

Confirmed: An episode of laboratory confirmed malaria parasitaemia in any person (symptomatic or asymptomatic). 


\section{Measles}

\section{(Measles virus) (Irish definition)}

\section{Clinical description}

Clinical picture compatible with measles i.e. a generalised erythematous rash lasting $>3$ days and a temperature $>38^{\circ} \mathrm{C}$ and one or more of the following cough, coryza (rhinitis), Koplik's spots or conjunctivitis.

\section{Laboratory criteria for diagnosis}

One of the following:

- Detection of measles IgM antibody in absence of recent vaccination

- Four fold or higher rise in measles IgG antibody level in absence of recent vaccination

- Detection of measles virus (not vaccine strains) in a clinical specimen

\section{Case classification}

Possible: Clinically compatible cases

Confirmed: A case that is laboratory confirmed or a clinically compatible case which is epidemiologically linked to a confirmed case. A laboratory-confirmed case does not need to meet the clinical case definition.

A measles case is epidemiologically linked if:

- There was exposure to a laboratory confirmed case during the infectious period (four days before to four days after rash onset) and

- This exposure occurred within the expected incubation period of the case under investigation -7 to 18 days (mean 14 days) before rash onset. 


\section{Meningococcal Disease \\ (Neisseria meningitidis) (Irish definition)}

\section{Clinical description}

Clinical picture compatible with meningococcal disease e.g. meningitis and/or septicaemia that may progress rapidly to purpura fulminans, shock or death. Other manifestations are possible.

\section{Case classification}

A 'Possible' case of meningococcal infection includes children or adults who have:

- Evidence of acute sepsis, with or without meningitis, together with characteristic haemorrhagic purpura

- Clinical evidence of sepsis without a purpuric rash, in whom no other cause of sepsis is identified, and in whom meningococcus is isolated from an eye, throat or nasal swab

- Received pre-admission antibiotics, have laboratory evidence of bacterial meningitis but are culture negative

A 'Presumed' case of meningococcal infection includes children or adults who have:

- Gram negative intracellular diplococcus detected in cerebrospinal fluid on microscopy

- Meningococcus isolated from an eye, throat or nasal swab, together with characteristic purpuric rash

- Meningococcus isolated from an eye, throat or nasal swab and who have clinical and laboratory features of bacterial meningitis (cerebrospinal fluid pleocytosis) in whom no other cause of meningitis is identified

- A clinically compatible illness and who have gram negative intracellular diplococci detected in skin scrapings taken from the characteristic haemorrhagic rash

- A clinically compatible illness with a serological response which is reported by a reference laboratory as consistent with recent acute infection

A 'Definite' case of meningococcal infection includes children or adults who have:

- Neisseria meningitidis isolated from blood, cerebrospinal fluid or other normally sterile body site (e.g. blood, synovial fluid, pleural or pericardial fluid) or from a petechial or purpuric lesion

- a positive PCR test for meningococcus obtained on blood, cerebrospinal fluid or specimen from another sterile site 


\section{Mumps \\ (Mumps virus) (EU)}

\section{Clinical description}

Clinical picture compatible with mumps, i.e. acute onset of unilateral or bilateral tender, self-limiting swelling of the parotid or other salivary gland, lasting $>2$ days, and without other apparent cause.

\section{Laboratory criteria for diagnosis}

One of the following:

- Detection of mumps IgM antibody

- Demonstration of specific mumps antibody response in absence of recent vaccination

- Isolation of mumps virus (not vaccine strains) from clinical specimen

- Detection of mumps nucleic acid

\section{Case classification}

Possible: A case that meets the clinical case definition ${ }^{\star \star}$

Probable: A case that meets the clinical case definition and is epidemiologically linked* to a confirmed case

Confirmed: A case that is laboratory confirmed.

** This definition of a possible case has been added to the EU definition

* See General Principles for the Application of these Case Definitions page 5 


\section{Non-specific urethritis}

\section{Clinical description}

Urethral inflammation that is not the result of infection with Neisseria gonorrhoeae.

\section{Laboratory criteria for diagnosis}

No evidence of $N$. gonorrhoeae infection by culture, gram stain, or antigen or nucleic acid detection

\section{Case classification}

Possible: N/A

Probable: N/A

Confirmed: A clinically compatible case in a male in whom gonorrhoea is not found, either by culture, gram stain, or antigen or nucleic acid detection. 


\section{Noroviral infection}

\section{(Norovirus)}

\section{Clinical description}

Clinical picture compatible with an acute gastrointestinal illness, usually characterised by sudden onset of vomiting and/or diarrhoea. Other symptoms may include abdominal cramps and a flu-like illness.

\section{Laboratory criteria for diagnosis}

One of the following:

- Norovirus detected in faeces by electron microscopy

- Detection of virus-specific RNA in faeces

\section{Case classification}

Possible: N/A

Probable: A clinically compatible case with an epidemiological link* to a confirmed case

Confirmed: A clinically compatible case that is laboratory confirmed.

* See General Principles for the Application of these Case Definitions page 5 


\section{Paratyphoid \\ (Salmonella paratyphi) (see typhoid)}




\section{Pertussis}

\section{(Bordetella pertussis) (EU)}

\section{Clinical description}

Clinical picture compatible with pertussis i.e. a cough lasting at least 2 weeks with one of the following: paroxysms of coughing, inspiratory "whoop", or post-tussive vomiting, without other apparent cause.

\section{Laboratory criteria for diagnosis}

One of the following:

- Demonstration of a specific pertussis antibody response in absence of recent vaccination

- Detection of nucleic acid

- Isolation of $B$. pertussis from clinical specimen

\section{Case classification}

Possible: $\quad$ A case that meets the clinical case definition

Probable: A case that meets the clinical case definition and has an epidemiological link*

Confirmed: A case that is laboratory confirmed

* See General Principles for the Application of these Case Definitions page 5 


\section{Plague \\ (Yersinia pestis) (EU)}

\section{Clinical description}

The disease is characterised by fever, chills, headache, malaise, prostration, and leukocytosis that manifests in one or more of the following principal clinical forms:

- Regional lymphadenitis (bubonic plague)

- Septicaemia without an evident bubo (septicaemic plague)

- Plague pneumonia

- Pharyngitis and cervical lymphadenitis.

\section{Laboratory criteria for diagnosis}

One of the following:

- Isolation of Y. pestis from a clinical specimen

- Demonstration of a specific antibody response to Y. pestis F1 antigen (Four fold or greater rise) ${ }^{\star *}$

For probable case - one of the following

- Elevated serum antibody titre(s) to Y. pestis fraction 1 (F1) antigen (without documented specific change) in a patient with no history of plague vaccination

- Detection of F1 antigen in a clinical specimen by fluorescent assay

\section{Case classification}

Possible: A clinically compatible case

Probable: A clinically compatible case with "probable" laboratory results Confirmed: A clinically compatible case with confirmatory laboratory results.

** The description in the EU case definition document has been elaborated upon here. 


\section{Q Fever \\ (Coxiella burnetii) (EU)}

\section{Clinical description}

A febrile illness accompanied by rigors, myalgia, malaise and retrobulbar headache. Severe disease can include acute hepatitis, pneumonia, meningoencephalitis and abortion. Clinical laboratory findings may include elevated liver enzyme levels and abnormal film findings.

\section{Laboratory criteria for diagnosis}

- Isolation of $C$. burnetii from a clinical specimen

- Demonstration of a specific antibody response by a four fold increase in specific antibody titre

- Demonstration of $C$. burnetii in a clinical specimen by detection of antigen or nucleic acid

For probable case

A single high titre of specific antibodies

\section{Case classification}

Possible: N/A

Probable: A clinically compatible case that fulfils the laboratory criteria for a probable case or has an epidemiological link ${ }^{*}$

Confirmed: A laboratory confirmed case that is clinically compatible or has an epidemiological link*.

* See General Principles for the Application of these Case Definitions page 5 


\section{Rabies}

\section{(Rabies virus) (EU)}

\section{Clinical description}

Rabies is an acute encephalomyelitis that almost always progresses to coma or death within 10 days after the first symptom.

\section{Laboratory criteria for diagnosis}

One of the following:

- Detection by direct fluorescent antibody of viral antigens in a clinical specimen (preferably the brain or the nerves surrounding hair follicles in the nape of the neck)

- Isolation (in cell culture or in a laboratory animal) of rabies virus from saliva, cerebrospinal fluid, or central nervous system tissue

- Identification of a rabies-neutralising antibody titre (complete neutralisation) in the serum or cerebrospinal fluid of an unvaccinated person

- Detection of rabies nucleic acid in clinical specimen

\section{Case classification}

Possible: A clinically compatible case without laboratory confirmation Probable: N/A

Confirmed: A clinically compatible case that is laboratory confirmed. 


\section{Rubella}

\section{(Rubella virus) (EU)}

\section{Clinical description}

Clinical picture compatible with rubella, i.e. acute onset of generalised maculopapular rash and arthralgia/arthritis, lymphadenopathy or conjunctivitis.

\section{Laboratory criteria for diagnosis}

One of the following:

- Detection of rubella IgM antibody in the absence of recent vaccination

- Demonstration of a specific rubella antibody response in absence of recent vaccination (four fold or greater rise*)

- Isolation of rubella virus in the absence of recent vaccination

- Detection of rubella nucleic acid in clinical specimen

\section{Case classification:}

Possible: A case that meets the clinical case definition

Probable: A clinically compatible case that has an epidemiological link*

Confirmed: A clinically compatible case that is laboratory confirmed.

\section{Rubella (congenital)}

\section{Clinical description}

An illness usually manifesting in infancy resulting from rubella infection in utero and characterised by signs or symptoms from the following categories:

a) Cataracts/congenital glaucoma, congenital heart disease (most commonly patent ductus arteriosus, or peripheral pulmonary artery stenosis), loss of hearing, pigmentary retinopathy

b) Purpura, splenomegaly, jaundice, microcephaly, mental retardation, meningoencephalitis, radiolucent bone disease

\section{Laboratory criteria for diagnosis}

One of the following:

- Isolation of rubella virus

- Demonstration of rubella-specific lgM antibody in the infant

- Infant rubella antibody level that persists at a higher level and for a longer period than expected from passive transfer of maternal antibody (i.e. rubella titre that does not drop at the expected rate of a two fold dilution per month)

* See General Principles for the Application of these Case Definitions page 5 


\section{Case classification}

Possible: A case with some compatible clinical findings but not meeting the criteria for a probable case or any infant born to a mother with documented or suspected rubella infection in pregnancy

Probable: A case that is not laboratory confirmed and that has any two complications listed in paragraph a) of the clinical description or one complication from paragraph a) and one from paragraph b), and lacks evidence of any other aetiology

Confirmed: A clinically compatible case that is laboratory confirmed Infection only: a case that demonstrates laboratory evidence of infection, but without any clinical symptoms or signs. 


\section{Salmonellosis}

\section{(Salmonella enterica) (EU)}

\section{Clinical description}

Clinical picture compatible with salmonellosis e.g. diarrhoea, abdominal pain, nausea, and sometimes vomiting. Cases may also be asymptomatic. The organism may cause extraintestinal infections. ${ }^{* *}$

\section{Laboratory criteria for diagnosis}

Isolation of Salmonella sp. (non-typhi, non-paratyphi) from a clinical specimen

\section{Case classification}

Possible: N/A

Probable: A laboratory confirmed isolate without clinical information or a case with clinical symptoms that has an epidemiological link ${ }^{\star}$

Confirmed: A clinically compatible case that is laboratory confirmed.

** The description in the EU case definition document has been elaborated upon here.

* See General Principles for the Application of these Case Definitions page 5 


\section{Severe Acute Respiratory Syndrome \\ (SARS-associated coronavirus)}

The following case definitions (dated 23 January 2004) are based on EU and WHO definitions and apply to the surveillance of SARS in the absence of known transmission (person-to-person) worldwide. These case definitions focus on the identification of persons with severe unexplained pneumonia either returning from a country with previous documented transmission of SARS or who are part of a cluster within a health care facility in Ireland. These definitions will change in the event of re-emergence of SARS. Please consult NDSC website at www.ndsc.ie.

\section{Clinical case definition}

The following case definition of SARS is consistent with the WHO clinical case definition and has been developed for public health purposes.

The respiratory illness usually will be severe enough to warrant hospitalisation and include a history of:

Fever of $38^{\circ} \mathrm{C}$ (documented or reported)

\section{AND}

One or more symptoms of lower respiratory tract illness (cough, difficulty breathing, shortness of breath)

\section{AND}

Radiographic evidence of lung infiltrates consistent with pneumonia or respiratory distress syndrome (RDS) or autopsy findings consistent with the pathology of pneumonia or RDS without an identifiable cause.

\section{AND}

No alternative diagnosis to fully explain the illness.

It is important that clinicians obtain a detailed travel history from patients with symptoms and signs consistent with clinical SARS as 
well as ascertain whether other family members and/or close contacts (particularly within the hospital setting) have had a similar illness within the $\mathbf{1 0}$ days prior to the patient's onset of illness.

\section{Case classification}

\section{Possible case:}

(a) Individual case

A person fulfilling the clinical case definition of SARS

\section{AND}

within ten days of onset of illness, a history of travel to an area classified by WHO as a potential zone of re-emergence of SARS (this includes an area identified as the source of the November 2002 outbreak and/or an area with increased likelihood of animal to human transmission of SARS-CoV infection).

(b) Health care worker $(\mathrm{HCW})$ cluster

Two or more HCWs in the same health care facility fulfilling the clinical case definition of SARS (see above) and with onset of illness within the same 10-day period. The definition of the health care unit in which the cluster occurs will depend on the local situation. Unit size may range from an entire health care facility if small, to a single department or ward of a large tertiary hospital.

(c) Other hospital cluster

Hospital acquired illness in three or more persons (health care workers and/or other hospital staff and/or patients and/or visitors) in (or linked to) the same health care unit fulfilling the clinical case definition of SARS (see above) and with onset of illness within the same 10-day period.

In order to detect a cluster of SARS in the healthcare setting, clinicians are asked to notify cases of unexplained pneumonia in healthcare workers to the Medical Officer of Health (Director of Public Health). All healthcare workers should be asked to inform the Occupational Health Physician/Department if they develop signs of severe pneumonia that require hospital admission.

\section{Probable case:}

(a) An individual with symptoms and signs consistent with clinical SARS (possible case) and with preliminary laboratory evidence of SARS-CoV based on the following: 


\section{Either}

Single positive antibody test for SARS-CoV

\section{Or}

Positive PCR for SARS-CoV on a single clinical specimen and assay

\section{(b) Laboratory acquired probable case}

An ill person who has developed the symptoms reported below in the 10 days following working with or having been in contact with a laboratory activity involved with SARS-CoV manipulation:

- Fever $\left(>38^{\circ} \mathrm{C}\right)$

- One or more respiratory symptoms including cough, difficulty breathing, shortness of breath

\section{And}

- Radiographic evidence of lung infiltrates consistent with pneumonia or respiratory distress syndrome.

This person should be considered as a SARS probable case and managed accordingly until the contrary is proven.

\section{Confirmed case:}

An individual with symptoms and signs consistent with clinical SARS (possible case) and with laboratory evidence of SARS-CoV infection based on one or more of the following:

\section{(a) PCR positive for SARS-CoV using a validated method from:}

At least two different clinical specimens (e.g. nasopharyngeal and stool)

Or

The same clinical specimen collected on two or more occasions during the course of the illness (e.g. sequential nasopharyngeal aspirates)

\section{Or}

Two different assays or repeat PCR using a new RNA extract from the original clinical sample on each occasion of testing.

\section{(b) Seroconversion by ELISA or IFA}

Negative antibody test on acute serum followed by positive antibody test on convalescent phase serum tested in parallel 


\section{Or}

Four fold or greater rise in antibody titre between acute and convalescent phase sera tested in parallel.

\section{(c) Virus isolation}

Isolation in cell culture of SARS-CoV from any specimen and PCR confirmation using a validated method.

Testing should only be undertaken in a national or regional reference laboratory as per WHO recommendations. 


\section{Shigellosis \\ (Shigella sp.) (EU)}

\section{Clinical description}

An illness of variable severity characterised by diarrhoea, fever, nausea, cramps, and tenesmus.

\section{Laboratory criteria for diagnosis}

Isolation of Shigella sp. from a clinical specimen

\section{Case classification}

Possible: N/A

Probable: A clinically compatible case with an epidemiological link ${ }^{*}$

Confirmed: A clinically compatible case that is laboratory confirmed.

* See General Principles for the Application of these Case Definitions page 5 


\section{Smallpox \\ Nariola virus) (EU)}

\section{Clinical description}

An illness with acute onset of fever over $38^{\circ} \mathrm{C}$ followed by a rash characterised by vesicles or firm pustules at the same stage of development without other apparent cause and with a predominantly centrifugal distribution.

Atypical presentations may include:

- haemorrhagic lesions

- flat velvety lesions not appearing as typical vesicles nor progressing to pustules.

Laboratory criteria for diagnosis

- Isolation of smallpox (variola) virus from a clinical specimen

- Polymerase chain reaction (PCR) identification of variola DNA in a clinical specimen, followed by sequencing

- Negative stain electron microscopy (EM) identification of variola virus in a clinical specimen

\section{Case classification}

Possible: A clinically compatible case

A case that has an atypical presentation and has an epidemiological link* to confirmed or probable cases

Probable: A clinically compatible case with either identification of orthopox virus by EM or PCR, or an epidemiological link* to other probable or confirmed cases

Confirmed: For an initial case, a clinically compatible case with laboratory confirmation by EM and PCR, followed by sequencing.

During an outbreak, a clinically compatible case with an epidemiological link ${ }^{*}$ and, where possible, laboratory confirmation by either EM or PCR.

* See General Principles for the Application of these Case Definitions page 5 


\section{Staphylococcal food poisoning \\ (Enterotoxigenic Staphylococcus aureus)}

\section{Clinical description}

A person with an acute gastrointestinal illness, characterised by abrupt onset of nausea, abdominal cramps, vomiting +/- diarrhoea.

\section{Laboratory criteria for diagnosis}

One of the following:

- Demonstration of staphylococcal enterotoxin in epidemiologically implicated food

- Isolation of S. aureus with the same phage type from faeces or vomitus of two or more epidemiologically linked ill persons

- Isolation of $10^{5}$ colony-forming units of $S$. aureus from epidemiologically implicated food

\section{Case classification}

Possible: N/A

Probable: A clinically compatible case with an epidemiological link ${ }^{*}$

Confirmed: A clinically compatible case that is laboratory confirmed.

* See General Principles for the Application of these Case Definitions page 5 


\section{Staphylococcus aureus bacteraemia}

\section{(S. aureus (blood))}

\section{Laboratory criteria for diagnosis}

Isolation of $S$. aureus from blood culture. The isolate should be identified as $S$. aureus by producing a positive coagulase test, preferably tube coagulase. Methicillin susceptibility should be determined using one or more of the following methods:

- Oxacillin screen plate, using National Committee for Control of Laboratory Standards (NCCLS) methodology (preferred routine method)

- Oxacillin (or methicillin) disc susceptibility testing, using NCCLS, British Society of Antimicrobial Chemotherapy (BSAC) or other internationally recognised methodology

- Detection of altered penicillin binding protein (PBP2a)

- Detection of the gene encoding methicillin resistance (mecA)

\section{Case classification}

Possible: N/A

Probable: N/A

Confirmed: A case that is laboratory confirmed. 


\section{Streptococcus group A infection (invasive)}

(S. pyogenes (blood, CSF or other normally sterile site))

\section{Clinical description}

An acute febrile illness which may be associated with Streptococcal Toxic Shock Syndrome (STSS). STSS is characterised by hypotension (fifth percentile of systolic blood pressure in children, or $<90 \mathrm{mmHg}$ systolic pressure in adolescents and adults) and two or more of the following:

- Renal impairment (creatinine greater than twice upper limit of normal for age)

- Coagulopathy (platelets $<100,000 \times 10^{6} / \mathrm{l}$ or evidence of disseminated intravascular coagulation)

- Liver dysfunction (ALT, AST or bilirubin more than twice upper limit of normal for age)

- Adult respiratory distress syndrome (pulmonary infiltrates and hypoxaemia without cardiac failure or generalised oedema)

- Generalised erythematous rash that may desquamate

- Soft tissue necrosis (necrotising fasciitis, myositis, gangrene).

\section{Laboratory criteria for diagnosis}

Isolation of group A streptococcus (S. pyogenes) from a normally sterile site (e.g. blood, cerebrospinal fluid, pleural fluid)

For probable case (STSS only)

Isolation of group A streptococcus from a nonsterile site (e.g. throat, sputum, vagina)

\section{Case classification}

Possible:

$\mathrm{N} / \mathrm{A}$

Probable (STSS): A clinically compatible case and meeting the probable laboratory criteria

Confirmed: A case that is laboratory confirmed. 


\section{Streptococcus pneumoniae infection (invasive)}

(S. pneumoniae (blood, CSF or other normally sterile site)) (EU)

\section{Clinical description}

S. pneumoniae causes many clinical syndromes, depending on the site of infection (e.g. acute otitis media, pneumonia, bacteraemia, or meningitis).

\section{Laboratory criteria for diagnosis}

One of the following:

- Isolation of S. pneumoniae from a normally sterile site (e.g., blood, cerebrospinal fluid, or, less commonly, joint, pleural, or pericardial fluid)

- Detection of S. pneumoniae nucleic acid from a normally sterile site

For probable case

- Detection of S. pneumoniae antigen from a normally sterile site

\section{Case classification}

Possible: A clinically compatible case without any laboratory confirmation or with identification from a non-sterile site

Probable: A clinically compatible case that is antigen positive

Confirmed: A clinically compatible case that is laboratory confirmed. 


\section{Syphilis \\ (Treponema pallidum) (EU)}

(See also Syphilis (congenital and neonatal))

\section{Syphilis, primary}

\section{Clinical description}

A stage of infection with T. pallidum characterised by one or more chancres (ulcers). Chancres might differ considerably in clinical appearance.

\section{Laboratory criteria for diagnosis}

One of the following:

- Detection of specific IgM by EIA

- Demonstration of T. pallidum in clinical specimens by darkfield microscopy, direct fluorescent antibody (DFA-TP), or equivalent methods

For probable case

A reactive serological test (nontreponemal: Venereal Disease Research Laboratory [VDRL] or rapid plasma reagin [RPR]; treponemal: fluorescent treponemal antibody absorbed [FTA-ABS] or microhaemagglutination assay for antibody to T. pallidum [MHA-TP]) or Treponema pallidum

Phytohaemagglutination assay (TPPA) ${ }^{\star *}$.

\section{Case classification}

Possible: N/A

Probable: A clinically compatible case with one or more ulcers (chancres) consistent with primary syphilis and any reactive serologic test

Confirmed: A clinically compatible case that is laboratory confirmed.

\section{Syphilis, secondary}

\section{Clinical description}

A stage of infection caused by T. pallidum and characterised by localised or diffuse mucocutaneous lesions, often with generalised lymphadenopathy. The primary chancre may still be present.

\section{Laboratory criteria for diagnosis}

Demonstration of T. pallidum in clinical specimens by darkfield microscopy, (DFA-TP), or equivalent methods

** The TPPA test is not mentioned in the EU definition. 
For probable case:

One of the following:

- A reactive serologic test (nontreponemal: Venereal Disease Research Laboratory [VDRL])

- Rapid plasma reagin [RPR]; treponemal: fluorescent treponemal antibody absorbed [FTA-ABS]

- Microhaemagglutination assay for antibody to T. pallidum [MHA-TP]

- Treponema pallidum phytohaemmaglutination assay (TPPA) ${ }^{\star *}$

\section{Case classification}

Possible: N/A

Probable: A clinically compatible case with any reactive serologic test

Confirmed: A clinically compatible case that is laboratory confirmed.

Syphilis, latent

\section{Clinical description}

A stage of infection caused by T. pallidum in which organisms persist in the body of the infected person without causing symptoms or signs.

\section{Laboratory criteria for diagnosis}

Demonstration of a positive reaction with a specific EIA but negative for laboratory test for infectious syphilis (see primary or secondary syphilis)

\section{Case classification}

Possible: N/A

Probable: No clinical signs or symptoms of syphilis and a positive laboratory test as above

Confirmed: N/A.

\section{Syphilis (congenital and neonatal)}

\section{Clinical description}

A condition caused by infection in utero with T. pallidum. A wide spectrum of severity exists, and only severe cases are clinically apparent at birth. An infant or child (aged <2 years) may have signs such as hepatosplenomegaly, rash, condyloma lata, snuffles, jaundice (nonviral hepatitis), pseudoparalysis, anemia, or edema (nephrotic syndrome and/or malnutrition). An older child may have stigmata (e.g., interstitial keratitis, nerve deafness, anterior bowing of shins, frontal bossing, mulberry molars, Hutchinson teeth, saddle nose, rhagades, or Clutton joints).

** The TPPA test is not mentioned in the EU definition. 


\section{Laboratory criteria for diagnosis}

Demonstration of T. pallidum by darkfield microscopy, fluorescent antibody, or other specific stains in specimens from lesions, placenta, umbilical cord, or autopsy material

\section{Case classification}

Probable: A condition affecting an infant whose mother had untreated or inadequately treated syphilis at delivery, regardless of signs in the infant, or an infant or child who has a reactive treponemal test for syphilis and any one of the following:

- Any evidence of congenital syphilis on physical examination

- Any evidence of congenital syphilis on radiographs of long bones

- A reactive cerebrospinal fluid (CSF) venereal disease research laboratory (VDRL)

- An elevated CSF cell count or protein (without other cause)

- A reactive fluorescent treponemal antibody absorbed-19S-IgM antibody test or IgM enzyme-linked immunosorbent assay

Confirmed: A case that is laboratory confirmed. 


\section{Tetanus}

\section{(Clostridium tetani) (EU)}

\section{Clinical description}

Clinical picture compatible with tetanus, i.e. acute onset of hypertonia and/or painful muscular contractions (usually of the muscles of the jaw and neck) and generalised muscle spasms without other apparent medical cause.

\section{Laboratory criteria for diagnosis}

One of the following:

- Detection of tetanus toxoid antibody in an unvaccinated and untreated patient

- Demonstration of a specific tetanus toxoid antibody response

\section{Case classification}

Possible: N/A

Probable: N/A

Confirmed: A clinically compatible case, with or without laboratory evidence. 


\section{Toxoplasmosis \\ (Toxoplasma gondii) (EU)}

\section{Clinical description}

A protozoan disease which presents with an acute illness with one or more of the following: Iymphadenopathy, encephalitis, chorioretinitis, dysfunction of the central nervous system. Congenital infections may also occur with hydrocephalus, microcephalus, intracerebral calcification, convulsions, cerebral retardation.

\section{Laboratory criteria for diagnosis}

One of the following:

- Demonstration of a specific toxoplasma antibody response

- Demonstration of the agent in body tissues or fluids or isolation in animals or cell culture

- Detection of toxoplasma nucleic acid

\section{Case classification}

Possible: N/A

Probable: N/A

Confirmed: A clinically compatible case that is laboratory confirmed.

\section{Toxoplasmosis (congenital)}

\section{Clinical description}

Congenital infection with T. gondii can result in a wide spectrum of clinical manifestations from asymptomatic or subclinical infection, aseptic meningitis in infancy, to severe multisystem involvement with hydrocephalus or microcephaly, chorioretinitis, thrombocytopenic purpura and hepatosplenomegaly. Children with congenital infection, even those with subclinical disease, are also at risk of later reactivation of chorioretinitis particularly toward the end of the first and into the second decade of life.

\section{Laboratory criteria for diagnosis}

One of the following:

- Histologic diagnosis of tachyzoites in infant tissues or body fluids

- Isolation of the parasite from an infant, usually from tissue obtained postmortem or on biopsy. (This requires animal or tissue culture inoculation and is not readily available).

- Detection of T. gondii by PCR testing of infant blood, CSF or tissue 


\section{Serologic testing.}

The serologic diagnosis of congenital toxoplasmosis is confounded both by the passive transfer of maternal $\lg G$ antibody and the fact that tests for toxoplasma specific lgM antibody can be negative in up to $50 \%$ of congenitally infected infants.

- Detection of toxoplasma specific lgM antibodies in cord blood or in the infant's blood is evidence of specific antibody synthesis by the infected infant in utero.

- Detection of toxoplasma specific IgA antibodies is also highly suggestive of infant infection.

Infant levels of toxoplasma specific IgG will normally equal or exceed those of the mother (due to active transfer of lgG by the placenta). However lgG levels that are four fold those of the mother are highly suggestive of infant infection and demonstration of rising lgG levels in sequential infant sera is conclusive evidence of congenital infection.

\section{Case classification}

Possible: Clinical symptoms compatible with congenital toxoplasmosis

Probable: Clinical symptoms suggestive of congenital toxoplasmosis with evidence of recent maternal infection (maternal toxoplasma IgM positive)

Confirmed: One of the following:

- Detection of T. gondii by PCR testing in the infant

- Presence of toxoplasma specific lgM

- Rising toxoplasma specific lgG in sequential infant sera. 


\section{Trichinosis \\ (Trichinella sp.) (EU)}

\section{Clinical description}

A disease caused by ingestion of trichinella larvae. The disease has variable clinical manifestations. Common signs and symptoms among symptomatic persons include eosinophilia, fever, myalgia and periorbital oedema.

\section{Laboratory criteria for diagnosis}

One of the following:

- Demonstration of trichinella larvae in tissue obtained by muscle biopsy

- Demonstration of a specific trichinella antibody response

\section{Case classification}

Possible: N/A

Probable: A clinically compatible case with an epidemiological link* Confirmed: A clinically compatible case that is laboratory confirmed.

* See General Principles for the Application of these Case Definitions page 5 


\section{Trichomoniasis \\ (Trichomonas vaginalis)}

\section{Clinical description}

A common and persistent protozoal disease of the genitourinary tract caused by Trichomonas vaginalis, characterised in women by vaginitis. The disease may cause a urethritis or cystitis but is frequently asymptomatic. In men the infectious agent invades and persists in the prostate, urethra or seminal vesicles and often causes only mild symptoms.

\section{Laboratory criteria for diagnosis}

Demonstration of the motile parasites either by microscopic examination of discharges or by culture, which is more sensitive.

\section{Case classification:}

Possible: N/A

Probable: N/A

Confirmed: A laboratory confirmed case. 


\section{Tuberculosis}

\section{(Mycobacterium tuberculosis complex) (EU)}

\section{Clinical criteria}

- A clinician's judgement that clinical and/or radiological signs and/or symptoms are compatible with tuberculosis

\section{and}

- A clinician's decision to treat the patient with a full course of anti-tuberculosis therapy.

\section{Laboratory criteria for diagnosis}

One of the following:

- Isolation of M. tuberculosis complex (except M. bovis BCG) from any clinical specimen by culture

- Evidence of acid-fast bacilli (AFB) at microscopic examination of spontaneous or induced sputum

\section{Case classification}

Classification according to laboratory criteria:

Other than definite: A case that meets the clinical criteria above but does not meet the laboratory criteria of a definite case.

Definite: A case with isolation of $M$. tuberculosis complex (except $M$. bovis BCG) from any clinical specimen. In countries where culture is not routinely available, a case with sputum smear examinations positive for AFB is also considered to be a definite case.

Classification according to the site of disease:

\section{Pulmonary tuberculosis}

Tuberculosis of the lung parenchyma or the tracheo-bronchial tree

\section{Extrapulmonary tuberculosis}

Tuberculosis affecting any site other than pulmonary as defined above

\section{Classification according to previous anti-tuberculosis treatment: Never treated}

A case who never received a treatment for active tuberculosis in the past or who received anti-tuberculosis drugs for less than one month.

\section{Previously treated}

A case who was diagnosed with active tuberculosis in the past and received anti-tuberculosis drugs (excluding preventive therapy) for at least one month. 


\section{Tularemia}

\section{(Francisella tularensis) (EU)}

\section{Clinical description}

Clinical picture compatible with one of the different forms of tularemia:

- Ulceroglandular (cutaneous ulcer with regional lymphadenopathy)

- Glandular (regional lymphadenopathy with no ulcer)

- Oculoglandular (conjunctivitis with preauricular lymphadenopathy)

- Oropharyngeal (stomatitis or pharyngitis or tonsillitis and cervical lymphadenopathy)

- Intestinal (intestinal pain, vomiting, and diarrhoea)

- Pneumonic (primary pneumonic disease)

- Typhoidal (febrile illness without early localising signs and symptoms).

\section{Laboratory criteria for diagnosis}

- Isolation of $F$. tularensis from a clinical specimen

- Demonstration of a specific antibody response

For probable case

- A single high titre

- Detection of $F$. tularensis in a clinical specimen by fluorescent assay

\section{Case classification}

Possible: N/A

Probable: A clinically compatible case that fulfils the laboratory criteria for a probable case or has an epidemiological link ${ }^{*}$

Confirmed: A clinically compatible case that is laboratory confirmed.

* See General Principles for the Application of these Case Definitions page 5 


\section{Typhoid/paratyphoid fever (Salmonella typhi/paratyphi) (EU)}

\section{Clinical description}

An illness caused by S. typhi or paratyphi that is often characterised by insidious onset of sustained fever, headache, malaise, anorexia, relative bradycardia, constipation or diarrhoea and non-productive cough. However, many mild and atypical infections occur.

\section{Laboratory criteria for diagnosis}

Isolation of S. typhi or paratyphi from blood, stool, or other clinical specimen

\section{Case classification}

Possible: N/A

Probable: A laboratory confirmed isolate without clinical information or a case with clinical symptoms with an epidemiological link*

Confirmed: A clinically compatible case that is laboratory confirmed.

* See General Principles for the Application of these Case Definitions page 5 


\section{Typhus \\ (Rickettsia prowazekii)}

\section{Clinical description}

An acute febrile illness usually associated with a rash.

\section{Laboratory criteria for diagnosis}

- A four fold or greater rise in rickettsial antibodies from paired sera

- A diagnostic rickettsial antibody titre from a single serum specimen

- Direct detection of rickettsia in a tissue sample, using immunohistochemistry or similar technique

- Detection of rickettsia-specific nucleic acids in blood or other tissue by PCR

For probable case

Positive Weil-Felix agglutination test

\section{Case classification}

Possible: N/A

Probable: A clinically compatible case that meets the probable laboratory criteria

Confirmed: A clinically compatible case that is laboratory confirmed. 


\section{Viral encephalitis}

\section{Clinical description}

Patients with viral encephalitis usually have signs and symptoms of meningeal irritation with alteration in consciousness. Focal neurological signs usually develop and seizures are common. Other manifestations may also be observed.

\section{Laboratory criteria for diagnosis}

One of the following:

- Detection of viral nucleic acid in CSF by PCR

- Culture of virus from CSF

- Specific antibody response between acute phase serum and convalescent serum

\section{Case classification}

Possible: N/A

Probable: N/A

Confirmed: Isolation of a viral species from the CSF. 


\section{Viral haemorrhagic fevers \\ (Lassa virus, Marburg virus, Ebola virus, Crimean-Congo haemorrhagic fever virus) (EU)}

\section{Lassa fever}

\section{Clinical description}

An illness of gradual onset with: malaise, fever, headache, sore throat, cough, nausea, vomiting, diarrhoea, myalgia, and chest pain. Haemorrhagic manifestations may follow.

\section{Laboratory criteria for diagnosis}

One of the following:

- Virus isolation

- Positive serology which may appear late in the course of the disease

- Positive skin biopsy (immunohistochemistry)

- Detection of Lassa virus nucleic acid

\section{Case classification}

Possible: N/A

Probable: A clinically compatible case with an epidemiological link*

Confirmed: A clinically compatible case that is laboratory-confirmed.

\section{Ebola/Marburg disease}

\section{Clinical description}

Begins with acute fever, diarrhoea that can be bloody and vomiting.

Headache, nausea, and abdominal pain are common. Haemorrhagic manifestations may follow. Some patients may also show a maculopapular rash on the trunk.

\section{Laboratory criteria for diagnosis}

One of the following:

- Positive virus isolation

- Positive skin biopsy (immunohistochemistry)

- Detection of Ebola/ Marburg virus nucleic acid

- Positive serology which may appear late in the course of the disease

* See General Principles for the Application of these Case Definitions page 5 


\section{Case classification}

Possible: N/A

Probable: A clinically compatible case with an epidemiological link*

Confirmed A clinically compatible case that is laboratory confirmed

\section{Crimean-Congo haemorrhagic fever}

\section{Clinical description}

An illness of gradual onset with acute high fever, chills, myalgia, nausea, anorexia, vomiting, headache and backache. Haemorrhagic manifestations may follow.

\section{Laboratory criteria for diagnosis}

One of the following:

- Virus isolation

- Detection of CCHF virus nucleic acid

- Positive serology which may appear late in the course of the disease

\section{Case classification}

Possible: N/A

Probable: A clinically compatible case with an epidemiological link*

Confirmed: A clinically compatible case that is laboratory confirmed.

* See General Principles for the Application of these Case Definitions page 5 


\section{Viral meningitis}

\section{Clinical description}

An acute illness with meningeal symptoms and fever.

\section{Laboratory criteria for diagnosis}

Raised cerebrospinal fluid white cell count and one of the following:

- Isolation of a viral pathogen from cerebrospinal fluid

- Detection of viral nucleic acid in cerebrospinal fluid

- Serological evidence of infection with a virus known to cause viral meningitis

For Probable case

Raised cerebrospinal fluid white cell count and isolation of a viral pathogen known to cause viral meningitis from a site other then cerebrospinal fluid

\section{Case Classification}

Probable: A clinically compatible case that meets the "probable" laboratory criteria but with no evidence of bacterial or fungal meningitis

Confirmed: A clinically confirmed case that is laboratory confirmed. 


\section{Yellow fever \\ (Yellow Fever virus) (EU)}

\section{Clinical description}

An illness characterised by acute onset and constitutional symptoms followed by a brief remission and a recurrence of fever, hepatitis, albuminuria, and, in some instances, renal failure, shock, and generalized haemorrhages.

\section{Laboratory criteria for diagnosis}

One of the following:

- Demonstration of a specific yellow fever antibody response in a patient who has no history of recent yellow fever vaccination and where cross-reactions to other flaviviruses have been excluded

- Virus isolation

- Detection of yellow fever antigen

- Detection of yellow fever nucleic acid

\section{Case classification}

Possible: N/A

Probable: A clinically compatible case with an epidemiological link*

Confirmed: Any clinically compatible case that is laboratory confirmed.

* See General Principles for the Application of these Case Definitions page 5 


\section{Yersiniosis}

\section{(Yersinia enterocolitica, Yersinia pseudo- tuberculosis) (EU)}

\section{Clinical description}

An illness of variable severity characterised by diarrhoea, fever, nausea, cramps and tenesmus.

\section{Laboratory criteria for diagnosis}

Isolation of $Y$. enterocolitica or pseudotuberculosis from a clinical specimen

\section{Case classification}

Possible: N/A

Probable: A clinically compatible case with an epidemiological link*

Confirmed: A case that is laboratory confirmed.

* See General Principles for the Application of these Case Definitions page 5 


\section{Outbreak}

An outbreak of infection or food-borne illness may be defined as two or more linked cases of the same illness or the situation where the observed number of cases exceeds the expected number, or a single case of disease caused by a significant pathogen (e.g. diphtheria or viral haemorrhagic fever). Outbreaks may be confined to some of the members of one family or may be more widespread and involve cases either locally, nationally or internationally. 


\section{Unusual cluster or changing pattern of illness}

A medical practitioner and a clinical director of a diagnostic laboratory are required to notify to the medical officer of health any unusual clusters or changing patterns of any illness, and individual cases thereof, that may be of public health concern.

An unusual cluster or changing pattern of illness means an aggregation of health events, grouped together in time or space, that is believed or perceived to be greater than could be expected by chance.

Features that should alert healthcare providers to the possibility of an unusual cluster or changing pattern of illness include:

- A rapidly increasing disease incidence in a normally healthy population

- An unusual increase in the number of people seeking care, particularly with fever, respiratory or gastrointestinal complaints

- An endemic disease rapidly emerging at an uncharacteristic time or in an unusual pattern (e.g. an increase in what appears to be chicken-pox like illness among adult patients, but which might be smallpox)

- Clusters of patients arriving from a single locale

- Large numbers of potentially fatal cases (e.g. a large number of acute flaccid paralysis with prominent bulbar palsies, suggestive of a release of botulinum toxin)

- Any one patient presenting with a disease that has bioterrorism potential (e.g. Anthrax, Smallpox, Botulism, Plague or Tularemia) 


\section{Appendix 1. \\ List of Notifiable Diseases}

\section{Disease}

Acute anterior poliomyelitis

Acute infectious gastroenteritis

Ano-genital warts

Anthrax

Bacillus cereus food-borne infection/intoxication

Bacterial meningitis (not otherwise specified)

Botulism

Brucellosis

Campylobacter infection

Chancroid

Chlamydia trachomatis infection (genital)

Cholera

Clostridium perfringens (type A) food-borne disease

Creutzfeldt Jakob disease

nv Creutzfeldt Jakob disease

Cryptosporidiosis

Diphtheria

Echinococcosis

Enterococcal bacteraemia

Enterohaemorrhagic Escherichia coli

Escherichia coli infection (invasive)

Giardiasis

Gonorrhoea

Granuloma inguinale

Haemophilus influenzae disease (invasive)

Hepatitis A (acute)

Hepatitis B (acute and chronic)

Hepatitis C

Herpes simplex (genital)

Influenza

Legionellosis

Leptospirosis

Listeriosis

Lymphogranuloma venereum

Malaria

\section{Causative Pathogen}

(Polio virus)

(Bacillus anthracis)

(B. cereus)

(Clostridium botulinum)

(Brucella sp.)

(Campylobacter sp.)

(Haemophilus ducreyi)

(C. trachomatis)

(Vibrio cholerae)

(C. perfringens)

(Cryptosporidium parvum)

(Corynebacterium

diphtheriae)

(Echinococcus sp.)

(Enterococcus sp. (blood))

(E. coli of serogroup

known to be toxin

producing)

(E. coli (blood, CSF))

(Giardia lamblia)

(Neisseria gonorrhoeae)

(H. influenzae (blood, CSF or other normally sterile site))

(Hepatitis A virus)

(Hepatitis B virus)

(Hepatitis C virus)

(Herpes simplex virus)

(Influenza A and B virus)

(Legionella sp.)

(Leptospira sp.)

(Listeria monocytogenes)

(Plasmodium falciparum, vivax, ovale, malariae) 
Measles

Meningococcal disease

Mumps

Non-specific urethritis

Noroviral infection

Paratyphoid

Pertussis

Plague

Q Fever

Rabies

Rubella

Salmonellosis

Severe Acute Respiratory Syndrome

Shigellosis

Smallpox

Staphylococcal food poisoning

Staphylococcus aureus bacteraemia

Streptococcus group A infection (invasive)

Streptococcus pneumoniae infection (invasive)

Syphilis

Tetanus

Toxoplasmosis

Trichinosis

Trichomoniasis

Tuberculosis

Tularemia

Typhoid

Typhus

Viral encephalitis

Viral meningitis

Viral haemorrhagic fevers

Yellow Fever

Yersiniosis
(Measles virus)

(Neisseria meningitidis)

(Mumps virus)

(Norovirus)

(Salmonella paratyphi)

(Bordetella pertussis)

(Yersinia pestis)

(Coxiella burnetii)

(Rabies virus)

(Rubella virus)

(Salmonella enterica)

(SARS-associated

coronavirus)

(Shigella sp.)

(Variola virus)

(Enterotoxigenic

Staphylococcus aureus)

(S. aureus (blood))

(S. pyogenes (blood, CSF or other normally sterile site))

(S. pneumoniae (blood, CSF or other normally sterile site))

(Treponema pallidum)

(Clostridium tetani)

(Toxoplasma gondii)

(Trichinella sp.)

(Trichomonas vaginalis)

(Mycobacterium

tuberculosis complex)

(Francisella tularensis)

(Salmonella typhi)

(Rickettsia prowazekii)

(Lassa virus, Marburg virus, Ebola virus, Crimean-Congo haemorrhagic fever virus) (Yellow Fever virus) (Yersinia enterocolitica, Yersinia pseudotuberculosis) 


\section{Appendix 2. \\ Members of the Notifiable Diseases Sub-Committee of the Scientific Advisory Committee, NDSC}

Dr Lelia Thornton (Chair)

Dr Karina Butler

Ms Catherine Cosgrove

Mr Stephen Dempsey

Mr Martin Devine*

Dr Peter Harrington

Dr Derval Igoe (Secretary)

Professor Conor Keane

Sr Jane Murphy

Dr Olive Murphy**

Mr Noel Shanaghy*
Faculty of Public Health Medicine, Royal College of Physicians of Ireland

Faculty of Paediatrics, Royal College of Physicians of Ireland

Environmental Health Officers Association Academy of Medical Laboratory Science Environmental Health Officers Association Irish College of General Practitioners National Disease Surveillance Centre Royal College of Physicians of Ireland Infection Control Nurses Association National Disease Surveillance Centre Academy of Medical Laboratory Science

* Mr Martin Devine and Mr Noel Shanaghy resigned from the sub-committee due to work commitments and were replaced by Ms Catherine Cosgrove and Mr Stephen Dempsey respectively

${ }^{\star *}$ Dr Olive Murphy resigned the sub-committee in September 1999 due to change in work commitments. 


\section{Acknowledgements}

We wish to acknowledge the work done in preparation of this document by Dr. Emer Feely, Dr Helena Murray, Dr Patricia McDonald, Dr Rob Cunney, Ms Aline Brennan, Dr Sarah Gee and Mr Ajay Oza. 


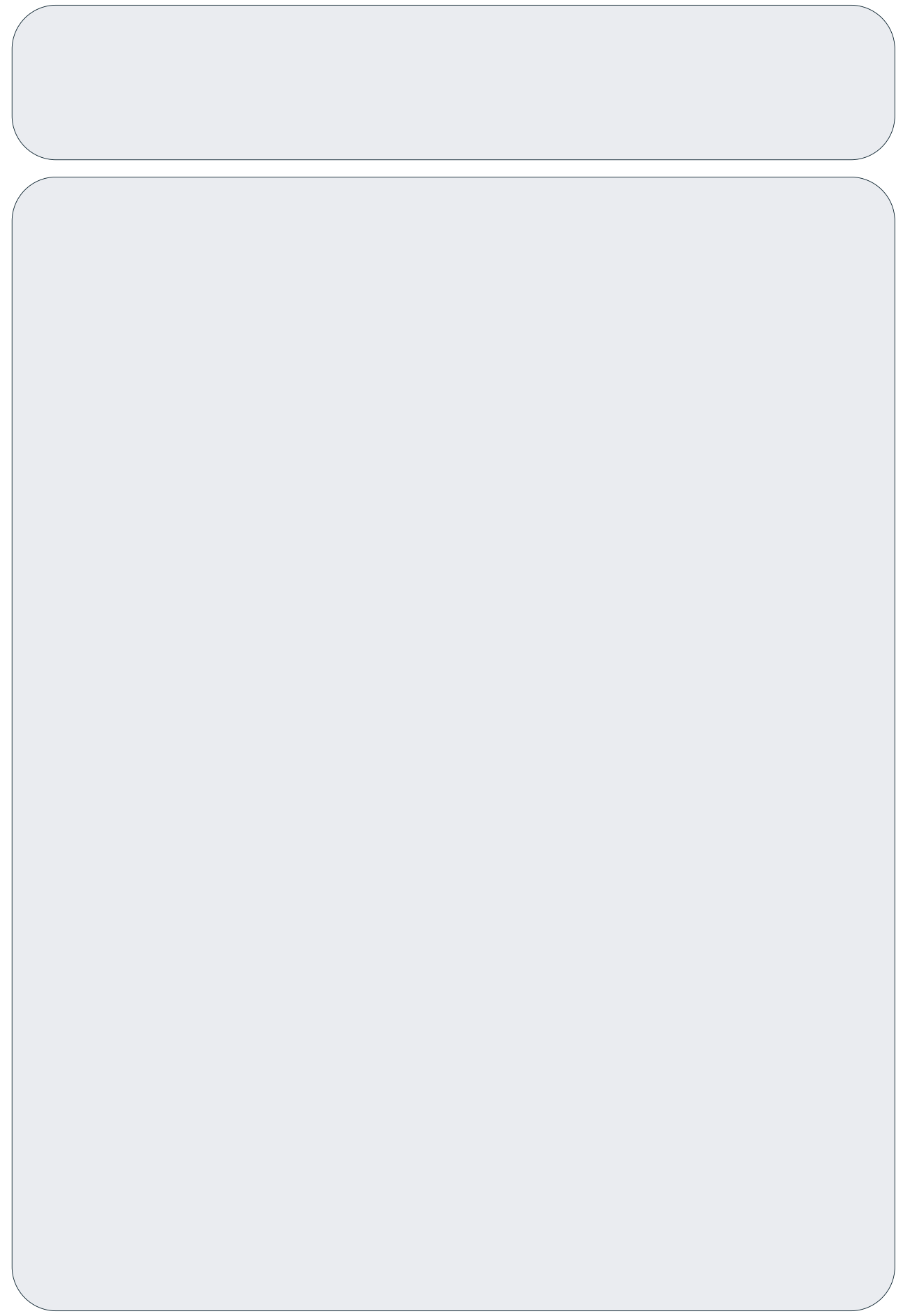

